\title{
Holographic trace anomaly at finite temperature
}

\author{
Bum-Hoon Lee \\ CQUeST, Sogang University, Seoul, Korea 121-742 \\ E-mail: bhl@sogang.ac.kr

\section{Siyoung Nam} \\ CQUeST, Sogang University, Seoul, Korea 121-742 \\ E-mail: stringphy@gmail.com \\ Chanyong Park \\ CQUeST, Sogang University, Seoul, Korea 121-742 \\ E-mail: cyong21@sogang.ac.kr
}

Department of Physics, Sogang University, Seoul, Korea 121-742 and

\begin{abstract}
We find an exact coordinate transformation rule from the $A d S_{5}$ Schwarzschild black hole in the Poincare and the global patch to the Fefferman-Graham coordinate system. Using these results, we evaluate the corresponding holographic stress tensor and trace anomaly of the boundary theory as a function of the radial coordinate. Following the AdS/CFT correspondence, we reinterpret the radial coordinate dependence of the trace anomaly as the Wilsonian renormalization $\operatorname{group}(\mathrm{RG})$ flow of the boundary theory.
\end{abstract}




\section{Contents}

1. Introduction 1

2. Constructing Finite Boundary Stress Tensor 3

3. Trace Anomaly in Schwarzschild-AdS Black Hole 6

3.1 Anomaly in the Poincare Patch 6

3.2 Anomaly in the Global Patch 9

4. Discussion 14

\section{Introduction}

The idea of the AdS/CFT correspondence [1, 2, 3](For reviews, see [四, 5]) is that string theory or M-theory in the near-horizon geometry of a collection of coincident D-branes or M-branes is equivalent to the low-energy world-volume theory of the corresponding branes. For example, the AdS/CFT correspondence relates type IIB superstring theory or M-theory in space-time geometries that are asymptotically anti-de Sitter(AAdS) times a compact space to conformal field theories(CFTs). The conjectured AdS/CFT correspondence can be regarded as a realization of the holographic principle [6, 7] and offer an example of a gravity/gauge theory duality. The strong coupling regime of the quantum field theory(QFT) defined on a background geometry which is conformally related to the geometry at the boundary of the AAdS space corresponds to the weak coupling regime of the string theory and vice versa. This implies that, at least in principle, we can obtain information on one side of the duality by performing computations on the other side.

According to the AdS/CFT correspondence, for every bulk field there is a corresponding gauge invariant boundary operator. The explicit prescription of [2, 3] is equating a partition function of the classical gravity theory which is the low energy limit of the string theory

$$
Z_{S U G R A}\left[\phi_{0}\right]=\int_{\phi_{0}} \mathcal{D} \phi \exp \left(-S\left[\phi, g_{\mu \nu}\right]\right)
$$

to a generating functional of the dual CFT

$$
Z_{C F T}\left[\phi_{0}\right]=\left\langle\exp \int_{\text {boundary }} d^{d} x \phi_{0} \mathcal{O}\right\rangle
$$


where $\phi_{0}$ denotes the boundary value of the field $\phi$. For example, the boundary value of the gravitational field is the source of the stress tensor of the dual theory. However, the boundary of the $A d S$ spacetime locates at the spatial infinity and the Weyl factor will diverge at the boundary due to the infinite volume factor of the on-shell action. Thus, we must regularize the theory and introduce counter terms to properly remove the infinities. The counter terms which are local functionals of the intrinsic geometry of the boundary are chosen to diverge at the boundary in such a way as to cancel the bulk divergences. After that, we can construct the finite boundary stress tensor. This procedure is called as a 'counter term' subtraction method[9, 10, 11]. We can do this in the Poincare patch or the global patch of the five-dimensional Schwarzschild-AdS(SAdS) black hole.

On the other hand, we can also do this in the so-called Fefferman-Graham(FG) coordinates. According to [12], the AAdS metric near the boundary allows a perturbative $\operatorname{expansion}^{1}$

$$
d s^{2}=G_{\mu \nu} d x^{\mu} d x^{\nu}=\frac{l^{2}}{z^{2}} d z^{2}+\frac{l^{2}}{z^{2}} g_{i j}(x, z) d x^{i} d x^{j}
$$

where

$$
g_{i j}(x, z)=g_{i j}^{(0)}+g_{i j}^{(2)} z^{2}+g_{i j}^{(4)} z^{4}+\cdots+g_{i j}^{(d)} z^{d}+h_{i j}^{(d)} z^{d} \ln z^{2}+\cdots .
$$

This is the solution of the Einstein equation in the Fefferman-Graham coordinate system. Originally, Fefferman and Graham considered a solution of AAdS space-time with $\mathbf{S}^{d}$ boundary for even $d$, where the logarithmic term appears naturally. As will be shown, in the cases of SAdS black hole with the boundary topology $\mathbf{R} \times \mathbf{R}^{3}$ (in the Poincare patch) and $\mathbf{R} \times \mathbf{S}^{3}$ (in the global patch), there is no logarithmic term in (1.4) so we will drop the logarithmic term from now on. Actually, the Euclidean versions of the above boundaries are $\mathbf{S} \times \mathbf{S}^{3}$ and $\mathbf{S} \times \mathbf{R}^{3}$ in the global patch and the Poincare patch, respectively.

In this paper, we will evaluate the boundary stress tensor from the bulk gravity side using AdS/CFT and study the radial dependence of the stress tensor by adopting a Wilsonian renormaization group $(\mathrm{RG})$ perspective. This task will be undertaken by using the exact black hole solution in the FG coordinates for the Poicare and the global patch. From the point of view of the metric (1.3), the boundary conditions are imposed at some finite cut-off value $z=z_{0}$, not at $z=0$ (which would be a true boundary of AdS) [15]. Since the effective energy scale on the boundary theory is associated with the radial coordinate of the bulk space-time [1], the physical meaning of the cut-off $z_{0}$ is that it acts as a UV regulator in the boundary theory [2, 16]. The bulk diffeomorphism that induces a Weyl transformation on the boundary metric is reminiscent of renormalization $\operatorname{group}(\mathrm{RG})$ flow 10, 17, 18]. So when the boundary is located at a finite distance in the radial direction, the position

\footnotetext{
${ }^{1} i, j=0,1,2,3$ stands for the boundary coordinates and $\mu, \nu=0,1,2,3,4$ for the bulk coordinates. We also denote the spatial coordinates by $\mathrm{a}, \mathrm{b}=1,2,3$.
} 
of the boundary can be reinterpreted as an energy cut-off of the boundary theory in the Wilsonian RG sense, where the energy 'cut-off' means that we have to integrate out all higher energy modes than this cut-off scale.

For this Wilsonian RG interpretation of the boundary theory, we need a well-defined boundary metric up to overall scale corresponding to the conformal structure of the boundary theory 10, 17, 18]. In the usual $r$-coordinate, this conformal structure of the boundary theory is not clear but in the FG coordinate this is manifest. So although we know a metric in the $r$-coordinate, to investigate the structure of the boundary theory it is important to find the same solution in the FG coordinate. After finding this solution in the FG coordinate, we have to choose the boundary metric as $g_{i j}(x, z)$ to obtain the finite boundary stress tensor

$$
T_{i j} \equiv \frac{2}{\sqrt{-g}} \frac{\delta S}{\delta g^{i j}}
$$

When considering the boundary metric as $\gamma_{i j}(x, z)=\frac{l^{2}}{z^{2}} g_{i j}(x, z)$ [9], the boundary stress tensor diverges when $z \rightarrow 0$. From now on, we consider $g_{i j}(x, z)$ as a boundary metric.

In section 2 we give a brief review of the (minimal) counter term subtraction method. In section 3 we find an exact black hole solution in the FG coordinate and calculate the boundary energy-momentum tensor as a function of the bulk radial coordinate. Using these results, we can investigate the trace anomaly depending on the radial position of the boundary, which is reinterpreted as a Wilsonian RG cut-off. In section 4 we finish this paper with some discussion and conclusion.

\section{Constructing Finite Boundary Stress Tensor}

In this section, we briefly review the two methods how to construct the finite stress tensor of the dual boundary theory. One of them is the (minimal) counter term subtraction method and the other is the holographic renormalization.

First we start by discussing the counter term subtraction method. One of the interesting problems in general relativity is how to construct a local stress tensor of a given theory in the curved background. In [8], Brown and York(BY) suggested a quasi-local stress tensor, which is defined as the functional derivative of the on-shell action with respect to the boundary metric and introduced the reference frame to render the action finite. This is called as a 'background' subtraction method. According to the AdS/CFT correspondence, the quasi-local stress tensor corresponds to the expectation value of the stress tensor in the dual CFT. Balasubramanian and Kraus(BK) constructed the Brown-York tensor for asymptotically AdS space time with a minimal set of the possible counter term[9]. For convenience, we call this counter term substraction method as BK method .

Let us consider the five-dimensional space time $\mathcal{M}$ foliated by four-dimensional time like hyper surfaces $M_{z}$ homeomorphic to the boundary $\partial \mathcal{M}$. We choose such a coordinate 
system that $x^{i}$ denotes the coordinates tangential to the hyper surfaces and $z$ the normal coordinate. The total $A d S_{5}$ action with a minimal counter term action is

$$
\begin{gathered}
S=\frac{1}{2 \kappa^{2}} \int_{\mathcal{M}} d^{5} x \sqrt{-G}\left(\mathrm{R}+\frac{12}{l^{2}}\right)-\frac{1}{\kappa^{2}} \int_{\partial \mathcal{M}} d^{4} x \sqrt{-\gamma} K \\
-\frac{3}{l \kappa^{2}} \int_{\partial \mathcal{M}} d^{4} x \sqrt{-\gamma}\left(1-\frac{l^{2}}{12} R^{(4)}\right)
\end{gathered}
$$

where $\kappa^{2}=8 \pi G$ and $G$ is the five-dimensional gravitational constant and $R^{(4)}$ is the curvature scalar constructed from the boundary metric $\gamma_{i j}$. The first term in the action is the ordinary Einstein-Hilbert action with a cosmological constant $-6 / l^{2}$ where $l$ is the curvature radius of the anti-de Sitter space. The second term is the Gibbons-Hawking boundary term defined by the trace of the extrinsic curvature

$$
K_{i j} \equiv-\nabla_{i} n_{j}
$$

which is needed for a well-defined variation of the action. The last term is the counter term which makes the action finite at the boundary. Then, the quasi-local stress tensor (or Brown-York tensor) $<T_{i j}^{B Y}>$ is given by

$$
\begin{aligned}
<T_{i j}^{B Y}> & =\frac{2}{\sqrt{-\gamma}} \frac{\delta S}{\delta \gamma^{i j}} \\
& =\frac{1}{\kappa^{2}}\left(K_{i j}-K \gamma_{i j}-\frac{3}{l} \gamma_{i j}+\frac{l}{2} G_{i j}^{(4)}\right),
\end{aligned}
$$

where $G_{i j}^{(4)} \equiv R_{i j}^{(4)}-(1 / 2) R^{(4)} \gamma_{i j}$ is an Einstein tensor of the boundary theory. Let us consider the following AAdS metric

$$
d s^{2}=\frac{l^{2}}{z^{2}}\left(g_{i j} d x^{i} d x^{j}+d z^{2}\right)
$$

When the spatial boundary of the AAdS space time locates at $z=0$, the boundary metric $\gamma_{i j}=\left(l^{2} / z^{2}\right) g_{i j}$ has a double pole. So to obtain a well-defined stress tensor we have to redefine the boundary metric by an appropriate scale transformation as $g_{i j}=\left(z^{2} / l^{2}\right) \gamma_{i j}$. Then, the well-defined boundary energy-momentum tensor is given by

$$
T_{i j} \equiv \frac{2}{\sqrt{-g}} \frac{\delta S}{\delta g^{i j}}=\frac{l^{2}}{z^{2}} T_{i j}^{B Y},
$$

which gives a finite value at the boundary of the AAdS space time [20].

Next, we assume that the boundary is at some finite value $z=z_{0}$. From the UV-IR relation, the boundary position $z_{0}$ can be reinterpreted as an energy scale of the boundary theory $\Lambda=1 / z_{0}$. From the viewpoint of Wilsonian RG sense, this implies that the higher energy modes of the boundary theory are integrated out. Therefore, this geometric setting describes the physics of the boundary theory at the given energy scale $\Lambda$. Since inserting a 
scale can usually break the conformal symmetry, the conformal symmetry of the boundary theory at $z_{0}$ is also broken so that we expect that there exists a trace anomaly depending on $\Lambda$. As will be shown, the effect of this energy scale is encoded to the finite size effect in the global patch, where the boundary has a $\mathbf{R} \times \mathbf{S}^{3}$ topology. When considering the black hole, there is another scale corresponding to the black hole mass, which also break the conformal symmetry and appears as a thermal effect of the boundary theory.

We outline another method, 'holographic renormalization', to evaluate the holographic stress tensor in the FG coordinates 10, 11]. According to the AdS/CFT correspondence, the generating function of a conformal field theory is given in terms of the on-shell gravitational action on $\partial \mathcal{M}$, which is proportional to the volume $\operatorname{Vol}(\partial \mathcal{M})$. The volume $\operatorname{Vol}(\partial \mathcal{M})$ of any conformally compact manifold $\mathcal{M}$ is infinite. So an appropriate renormalization of $\operatorname{Vol}(\partial \mathcal{M})$ must be carried out. This procedure starts from regulating the theory. To do this, we restrict the range of the radial coordinate to $\rho \geq \rho_{0}$ and think the boundary as located at $\rho=\rho_{0} \cdot{ }^{2}$ Therefore, the regulated action is given by

$$
\begin{array}{r}
S_{r e g}=\frac{1}{2 \kappa^{2}} \int_{\rho \geq \rho_{0}} d^{5} x \sqrt{-G}(\mathrm{R}-2 \Lambda)-\frac{1}{\kappa^{2}} \int_{\rho=\rho_{0}} d^{4} x \sqrt{-\gamma} K \\
=\frac{l^{3}}{2 \kappa^{2}} \int d^{4} x \sqrt{-g_{(0)}}\left[\frac{-6}{\rho_{0}^{2}}+\frac{1}{2}\left(\operatorname{Tr}\left[g_{(0)}^{-1} g_{(2)} g_{(0)}^{-1} g_{(2)}\right]\right.\right. \\
\left.\left.-\operatorname{Tr}\left[g_{(0)}^{-1} g_{(2)}\right] \operatorname{Tr}\left[g_{(0)}^{-1} g_{(2)}\right]\right) \ln \rho_{0}+\mathcal{O}\left(\rho_{0}^{2}\right)\right] .
\end{array}
$$

After subtracting the divergences by the counter terms and removing the regulator, we obtain the holographically renormalized action

$$
\begin{aligned}
S_{r e n}=\lim _{\rho_{0} \rightarrow 0} \frac{l^{3}}{2 \kappa^{2}}[ & S_{r e g}-\int d^{4} x \sqrt{-g_{(0)}}\left\{\frac{-6}{\rho_{0}^{2}}+\frac{1}{2}\left(\operatorname{Tr}\left[g_{(0)}^{-1} g_{(2)} g_{(0)}^{-1} g_{(2)}\right]\right.\right. \\
& \left.\left.\left.-\operatorname{Tr}\left[g_{(0)}^{-1} g_{(2)}\right] \operatorname{Tr}\left[g_{(0)}^{-1} g_{(2)}\right]\right) \ln \rho_{0}\right\}\right]
\end{aligned}
$$

Then, the holographic stress tensor can be obtained by the following definition 10

$$
<T_{i j}^{0}>=\frac{2}{\sqrt{-g_{(0)}}} \frac{\partial S_{r e n}}{\partial g_{(0)}^{i j}},
$$

which becomes in terms of $g_{(0)}, g_{(2)}$ and $g_{(4)}$

$$
\begin{aligned}
<T_{i j}^{0}>= & \frac{l^{3}}{\kappa^{2}}\left[2 g_{i j}^{(4)}-\frac{1}{4}\left[\operatorname{Tr}\left[g_{(0)}^{-1} g_{(2)}\right] \operatorname{Tr}\left[g_{(0)}^{-1} g_{(2)}\right]-\operatorname{Tr}\left[g_{(0)}^{-1} g_{(2)} g_{(0)}^{-1} g_{(2)}\right]\right] g_{i j}^{(0)}\right. \\
& \left.-\left(g_{(2)} g_{(0)}^{-1} g_{(2)}\right)_{i j}+\frac{1}{2} \operatorname{Tr}\left[g_{(0)}^{-1} g_{(2)}\right] g_{i j}^{(2)}\right]
\end{aligned}
$$

\footnotetext{
${ }^{2}$ Here, we use the variable $\rho=z^{2}$ for the sake of the convenient calculation, because the expansion (1.4) contains only even powers of $z$.
} 
As will be shown with an explicit calculation in the next section, this holographic stress tensor corresponds to the leading term of the boundary stress tensor defined in (2.5),

$$
<T_{i j}^{0}>=\lim _{\rho_{0} \rightarrow 0} T_{i j}
$$

which implies that at $\rho_{0}\left(z_{0}\right) \rightarrow 0$ the higher order corrections like $g_{(6)}, g_{(8)}, \cdots$ are suppressed.

\section{Trace Anomaly in Schwarzschild-AdS Black Hole}

\subsection{Anomaly in the Poincare Patch}

In the Poincare patch, a black hole (or black brane) metric is given by

$$
d s^{2}=-f(r) d t^{2}+\frac{d r^{2}}{f(r)}+\frac{r^{2}}{l^{2}} d \vec{x}^{2},
$$

with $f(r)=r^{2} / l^{2}-m / r^{2}$. In the above equation,

$$
m \equiv \frac{2 \kappa^{2} M l^{3}}{3 V_{3}}
$$

is related to a black hole mass $M$ and a volume of the three dimensional space $V_{3}$. The black hole horizon $r_{h}=\left(m l^{2}\right)^{1 / 4}$ satisfies $f\left(r_{h}\right)=0$ and the black hole entropy proportional to the area at this horizon is given by

$$
S=\frac{m^{3 / 4} V_{3}}{4 G l^{3 / 2}}
$$

Using the surface gravity $\left.K \equiv(1 / 2) \partial_{r} f(r)\right|_{r=r_{h}}$, the Hawking temperature is given by

$$
T_{H} \equiv \frac{K}{2 \pi}=\frac{m^{1 / 4}}{\pi l^{3 / 2}}
$$

Note that when $m=0$ this black hole metric reduces to a metric for a pure AdS space-time.

To obtain a black hole solution in the FG coordinate system, we must evaluate the following integral

$$
\int \frac{d r}{\sqrt{f(r)}}=-\int \frac{l d z}{z},
$$

the $(\mathrm{d}+1)$-dimensional result of which is given by

$$
r(z)=l\left[\left(\frac{l}{z}\right)^{\frac{d}{2}}+\frac{m}{4 l^{d-2}}\left(\frac{z}{l}\right)^{\frac{d}{2}}\right]^{\frac{2}{d}} .
$$

In this paper, we are interested in the five-dimensional case $(d=4)$ and thus the relation becomes

$$
r(z)=\frac{l^{2}}{z} \sqrt{1+\frac{m z^{4}}{4 l^{6}}}
$$


Then, the black hole metric in the FG coordinates 19] is

$$
d s^{2}=-F(z) d t^{2}+G(z) d \vec{x}^{2}+\frac{l^{2}}{z^{2}} d z^{2}
$$

where

$$
F(z)=\frac{l^{2}}{z^{2}} \frac{\left(1-\frac{m z^{4}}{4 l^{6}}\right)^{2}}{\left(1+\frac{m z^{4}}{4 l^{6}}\right)} \text { and } \quad G(z)=\frac{l^{2}}{z^{2}}\left(1+\frac{m z^{4}}{4 l^{6}}\right) .
$$

From the coordinate transformation (3.7), we can easily find the relations among the functions $F(z), G(z)$, the lapse function $f(r)$, and the coordinate $r$ as a function of $z$ :

$$
F(z)=f(r(z)) \text { and } \quad G(z)=\frac{r(z)^{2}}{l^{2}} .
$$

The equation $F(z)=0$ gives a radius of the event horizon $z_{h}=\sqrt{2} l^{3 / 2} / m^{1 / 4}$, which is related to $r_{h}$ under the coordinate transformation. The entropy proportional to the area at the event horizon is given by

$$
S=\frac{V_{3}}{4 G} \sqrt{G\left(z_{h}\right)^{3}}=\frac{m^{3 / 4} V_{3}}{4 G l^{3 / 2}} .
$$

The Hawking temperature in the FG coordinate is given by

$$
T_{H}=\left.\frac{1}{4 \pi} \partial_{r} f(r)\right|_{r=r_{h}}=\left.\frac{1}{4 \pi l} \frac{\partial_{z} F(z)}{\partial_{z} \sqrt{G(z)}}\right|_{z=z_{h}}
$$

where $l \partial_{z} \sqrt{G(z)}$ is due to the coordinate transformation. From this formula, the Hawking temperature in the FG coordinate becomes

$$
T_{H}=\frac{m^{1 / 4}}{\pi l^{3 / 2}}
$$

which gives the same Hawking temperature, as expected. In the FG coordinate, we can easily obtain the thermodynamic quantities of the black hole by using (3.11) and (3.13), which gives the exactly same results as those in the usual $r$-coordinate, (3.3) and (3.4).

Now, we evaluate the boundary stress tensor and the trace of it. First we follow the BK-method using (2.5). Here, only the following counter term is needed

$$
S_{c t}=-\frac{1}{\kappa^{2}} \int d^{4} x \sqrt{-\gamma} \frac{3}{l}
$$

because the Einstein tensor of the boundary metric $\gamma_{i j}$ is trivially zero in the Poincare patch. Then, the boundary stress tensor is given by

$$
\begin{aligned}
\kappa^{2}\left\langle T_{00}\right\rangle & =\frac{3 m}{2 l^{3}} \cdot\left(\frac{4 l^{6}-m \rho^{2}}{4 l^{6}+m \rho^{2}}\right)^{2}, \\
\kappa^{2}\left\langle T_{a a}\right\rangle & =\frac{m}{2 l^{3}} \cdot\left(\frac{4 l^{6}+3 m \rho^{2}}{4 l^{6}-m \rho^{2}}\right) .
\end{aligned}
$$


This is an exact result describing the boundary stress tensor at the RG scale $\Lambda=1 / \sqrt{\rho}$ and at the finite temperature $T_{H}=\frac{m^{1 / 4}}{\pi l^{3 / 2}}$. The trace of the stress tensor is given by

$$
\kappa^{2}\left\langle T_{i}^{i}\right\rangle=\frac{24 m^{2} l \rho^{3}}{\left(4 l^{6}-m \rho^{2}\right)\left(4 l^{6}+m \rho^{2}\right)} .
$$

Next, we calculate the stress tensor by the holographic renormalization method. Using the formula (2.9) we get the result

$$
\kappa^{2}<T_{i j}^{0}>=\frac{m}{2 l^{3}} \operatorname{diag}(3,1,1,1)
$$

For comparison, we expand the exact results (3.15) and (3.16) near $\rho=0$

$$
\begin{aligned}
\kappa^{2}\left\langle T_{00}\right\rangle & =\frac{3 m}{2 l^{3}}-\frac{3 m^{2} \rho^{2}}{2 l^{9}}+\frac{3 m^{3} \rho^{4}}{4 l^{15}}+\mathcal{O}\left(\rho^{6}\right), \\
\kappa^{2}\left\langle T_{a a}\right\rangle & =\frac{m}{2 l^{3}}+\frac{m \rho^{2}}{2 l^{9}}+\frac{m^{3} \rho^{4}}{8 l^{15}}+\mathcal{O}\left(\rho^{6}\right), \\
\kappa^{2}\left\langle T^{i}{ }_{i}\right\rangle & =\frac{3 m^{2} \rho^{3}}{2 l^{11}}+\frac{3 m^{4} \rho^{7}}{32 l^{23}}+\mathcal{O}\left(\rho^{11}\right) .
\end{aligned}
$$

Then, as expected, the leading part of the stress tensor $\left\langle T_{i j}>\right.$ defined by the BK-method corresponds to the stress tensor $\left\langle T_{i j}^{0}>\right.$ acquired by the holographic renormalization. These leading terms are also calculated in 20]. Furthermore, we can also write the stress tensor in the compact form. Using the equations (3.13) and (3.16), the stress tensor is rewritten by

$$
<T_{i j}>=<T_{i}^{i}>C(\rho) g_{i j}
$$

where

$$
C(\rho)=\frac{l^{2}}{4 \rho}+\frac{l^{2}\left(1-4 \delta_{i 0} \delta_{j 0}\right)}{3 \pi^{4} T_{H}^{4} \rho^{3}} .
$$

It is easy to check that, in the limit $\rho \rightarrow 0,<T^{i}{ }_{i}>\sim \rho^{3}, C(\rho) \sim 1 / \rho^{3}$ and $g_{i j} \sim \rho^{0}$, so that the leading term of the stress tensor is independent of the radial coordinate $\rho$. Note that the stress tensor is proportional to the boundary metric. By following the method of [9], we can also calculate the mass

$$
M=\lim _{\rho \rightarrow 0} \frac{3 m V_{3}}{8 \kappa^{2} l^{9}}\left|m \rho^{2}-4 l^{6}\right|,
$$

which confirms the normalization of the black hole mass density (3.2) and agrees with the result in [9, 21]. According to the AdS/CFT correspondence, this stress tensor can be reinterpreted as that of the boundary theory. From the viewpoint of the boundary theory, the black hole mass $m$ corresponds to the temperature of the boundary theory with (3.13) and the radial coordinate $\rho$ is reinterpreted as an inverse energy scale $\Lambda=1 / \sqrt{\rho}$ of the 
boundary theory. Hence we can write the trace in terms of the boundary variables, $\Lambda$ and $T_{H}$

$$
\begin{aligned}
\kappa^{2} T_{i}^{i} & =\frac{24 \sqrt{\Lambda}}{\frac{16}{\pi^{8}}\left(\frac{\Lambda}{T_{H}^{4}}\right)^{4}-1} \\
& =\frac{3 \pi^{8} T_{H}^{8}}{2 \Lambda^{3 / 2}}\left[1+\frac{\pi^{8}}{16}\left(\frac{T_{H}^{4}}{\Lambda}\right)^{2}+\frac{\pi^{16}}{256}\left(\frac{T_{H}^{4}}{\Lambda}\right)^{4}+\cdots\right]
\end{aligned}
$$

Since the radial coordinate dependence of the quantities in the bulk theory can be considered as a Wilsonian RG flow of the boundary theory, $\Lambda$ is introduced as an energy scale of the Wilsonian RG flow in the boundary theory where the higher energy modes than $\Lambda$ are integrated out. At the UV fixed point $\rho=0$ where the trace anomaly is zero, the boundary stress tensor satisfy the Stefan-Boltzman law 19, 22

$$
\kappa^{2}\left\langle\mathrm{~T}_{00}\right\rangle=\frac{3 \pi^{4} l^{3}}{2} T_{H}^{4}
$$

When $m=0$, this SAdS black hole solution reduces to the pure AdS space-time solution with a boundary $\mathbf{R} \times \mathbf{R}^{3}$. As is well known, the gravity theory on the pure AdS space-time corresponds to the $\mathrm{N}=4 \mathrm{SYM}$ which is superconformal. Therefore, we can easily expect that there is no trace anomaly for $\mathrm{N}=4$ boundary SYM. In (3.16), for $m \rightarrow 0$ the trace of the boundary theory becomes zero which implies that the boundary theory is always conformal theory independent of the scale. For $m \neq 0$, this describes the finite temperature

field theory defined on the Hawking temperature $T_{H}=m^{1 / 4} /\left(\pi l^{3 / 2}\right)$. At the UV fixed point $\rho=0$, the trace anomaly of this boundary theory is zero so this theory is still conformal. When $\rho \neq 0$, the trace anomaly is not zero due to the thermal correction. This trace anomaly grows monotonically as $\rho$ goes to the black hole horizon $\rho_{h}=z_{h}$ and at the black hole horizon this trace anomaly diverges (see figure 1 ).

\subsection{Anomaly in the Global Patch}

A SAdS black hole solution in the global coordinate is given by the following expression

$$
d s^{2}=-f(r) d t^{2}+\frac{1}{f(r)} d r^{2}+r^{2} d \Omega_{3}^{2}
$$

where

$$
f(r)=1+\frac{r^{2}}{l^{2}}-\frac{m}{r^{2}}
$$

and $d \Omega_{3}^{2}=w_{a b} d x^{a} d x^{b}=d \theta^{2}+\sin ^{2} \theta d \phi^{2}+\cos ^{2} \theta d \psi^{2}$ is a metric for a unit three-sphere $S^{3}$. Here, $m$ is defined by

$$
m=\frac{2 \kappa^{2} M}{3 V_{S^{3}}}
$$




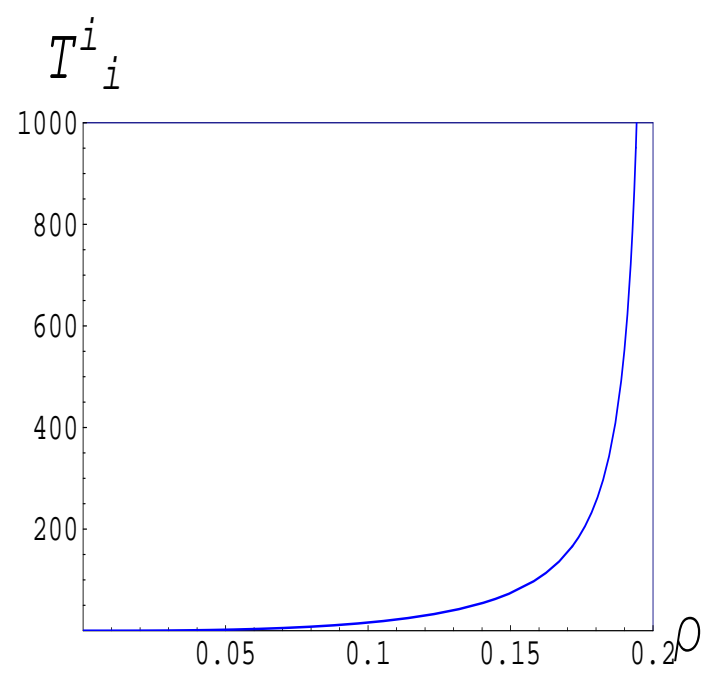

Figure 1: Trace Anomaly in the Poncare patch. For convenience, we set $l=1$ and $\kappa^{2}=1$. With $m=100$, the trace anomaly diverges at the black hole horizon $\rho_{h}=0.2$.

where $M$ is a black hole mass and $V_{S^{3}}=2 \pi^{2}$ is the volume of the unit three sphere. From the equation $f\left(r_{h}\right)=0$, the black hole horizon $r_{h}$ is given by

$$
r_{h}=l\left(\frac{\sqrt{1+\frac{4 m}{l^{2}}}-1}{2}\right)^{1 / 2}
$$

Then, the Hawking temperature is given by

$$
T_{H}=\frac{1}{2 \pi l} \cdot \sqrt{\frac{2+8 m / l^{2}}{\sqrt{1+4 m / l^{2}}-1}} .
$$

and the black hole entropy is

$$
S=\frac{\pi^{2}}{2 G} r_{h}^{3}=\frac{\pi^{2} l^{3}}{2 G}\left(\frac{\sqrt{1+\frac{4 m}{l^{2}}}-1}{2}\right)^{3 / 2} .
$$

To know the metric in the FG coordinate, we have to know the coordinate transformation between $r$ and $z$ by evaluating the integral (3.5), which is difficult to perform.

So instead of solving (3.5), we will find the metric in the FG coordinate by solving the Einstein equation with an appropriate metric ansatz. We make the following metric ansatz

$$
d s^{2}=-A(z) d t^{2}+B(z) d \Omega_{3}^{2}+\frac{l^{2}}{z^{2}} d z^{2} .
$$

Although this metric solution describes the same SAdS black hole in the usual $r$-coordinate system, it is a very important solution for the application of the AdS/CFT correspondence 
and the exact form is not known yet. To solve the Einstein equations exactly, we need two boundary conditions, one of which is that this solution is given by the pure AdS space-time near $z=0$. The other boundary condition will be fixed later. Using the ansatz (3.30) and the above boundary condition, we find an exact solution for the Einstein equation

$$
d s^{2}=l^{2}\left[-\frac{\left[\left(1-\frac{z^{2}}{4 l^{2}}\right)^{2}+c z^{4}+\frac{z^{2}}{2 l^{2}}-2\right]^{2}}{z^{2}\left[\left(1-\frac{z^{2}}{4 l^{2}}\right)^{2}+c z^{4}\right]} d t^{2}+\frac{d z^{2}}{z^{2}}+\frac{l^{2}\left[\left(1-\frac{z^{2}}{4 l^{2}}\right)^{2}+c z^{4}\right]}{z^{2}} d \Omega_{3}^{2}\right],
$$

where $c$ is an arbitrary constant which appears because of not imposing the other boundary condition. Since our solution is another representation of SAdS black hole solution in FG coordinate, $c$ must be identified by $m /\left(4 l^{6}\right)$ comparing with (3.24) which is the same as imposing the other boundary condition. Comparing two black hole solutions (3.24) and (3.31), we find that these two solutions are related by a coordinate transformation

$$
r(z)=\frac{l^{2}}{z} \sqrt{\left(1-\frac{z^{2}}{4 l^{2}}\right)^{2}+\frac{m z^{4}}{4 l^{6}}} .
$$

It is easy to check that the above equation (3.32) satisfies the coordinate transformation integral (3.5). Using this coordinate transformation, the functions, $A(z)$ and $B(z)$ are related to the lapse function in (3.24) and the coordinate transformation $r(z)$ :

$$
A(z)=f(r(z)) \text { and } B(z)=r(z)^{2} .
$$

In this coordinate, the black hole horizon is given by

$$
z_{h}=\frac{2 l}{\left(1+4 m / l^{2}\right)^{1 / 4}}
$$

which satisfy the equation $A\left(z_{h}\right)=0$. The black hole entropy is given by

$$
S=\frac{V_{S^{3}}}{4 G} \sqrt{B\left(z_{h}\right)^{3}}=\frac{\pi^{2} l^{3}}{2 G}\left(\frac{\sqrt{1+\frac{4 m}{l^{2}}}-1}{2}\right)^{3 / 2} .
$$

The Hawking temperature in the FG coordinate is given by

$$
T_{H}=\left.\frac{1}{4 \pi} \frac{\partial_{z} A(z)}{\partial_{z} \sqrt{B(z)}}\right|_{z_{h}}=\frac{1}{2 \pi l} \cdot \sqrt{\frac{2+8 m / l^{2}}{\sqrt{1+4 m / l^{2}}-1}} .
$$

Using these formula for the FG coordinate, we can easily calculate the thermodynamic properties of the SAdS black hole which are exactly the same as those obtained in the usual $r$-coordinate.

Now, we turn our attention to evaluating the boundary stress tensor, which can be done conveniently in the following metric, transformed by $\rho=z^{2}$,

$$
d s^{2}=-A(\rho) d t^{2}+\frac{l^{2} d \rho^{2}}{4 \rho^{2}}+B(\rho) d \Omega_{3}^{2}
$$


where

$$
\begin{aligned}
& A(\rho)=\frac{l^{2}}{\rho} \frac{\left[-2+\frac{\rho}{2 l^{2}}+\frac{m \rho^{2}}{4 l^{6}}+\left(1-\frac{\rho}{4 l^{2}}\right)^{2}\right]^{2}}{\left[\frac{m \rho^{2}}{4 l^{6}}+\left(1-\frac{\rho}{4 l^{2}}\right)^{2}\right]}, \\
& B(\rho)=\frac{l^{4}}{\rho}\left[\frac{m \rho^{2}}{4 l^{6}}+\left(1-\frac{\rho}{4 l^{2}}\right)^{2}\right] .
\end{aligned}
$$

Then, it is straightforward to read off each term in the FG expansion of the metric (3.37) and we specify some leading terms here :

$$
\begin{aligned}
g_{i j}^{(0)}= & \operatorname{diag}\left(-1, l^{2}, l^{2} \sin ^{2} \theta, l^{2} \cos ^{2} \theta\right), \\
g_{i j}^{(2)}= & \operatorname{diag}\left(-\frac{1}{2 l^{2}},-\frac{1}{2},-\frac{1}{2} \sin ^{2} \theta,-\frac{1}{2} \cos ^{2} \theta\right), \\
g_{i j}^{(4)}= & \operatorname{diag}\left(-\frac{l^{2}-12 m}{16 l^{6}}, \frac{l^{2}+4 m}{16 l^{4}}, \frac{l^{2}+4 m}{16 l^{4}} \sin ^{2} \theta, \frac{l^{2}+4 m}{16 l^{4}} \cos ^{2} \theta\right), \\
& \vdots
\end{aligned}
$$

Note that the perturbative expansion of (3.37) has no logarithmic term.

Now, we evaluate the exact boundary stress tensor at finite temperature, which is described by the black hole mass $m$ in the bulk, with the RG scale $\Lambda$ related to the inverse of the boundary position $1 / \sqrt{\rho}$. From (2.5), the boundary stress tensor is given by

$$
\begin{aligned}
& \kappa^{2}\left\langle T_{00}\right\rangle=\frac{3\left(l^{2}+4 m\right)}{8 l^{3}} \cdot\left(\frac{-16 l^{6}+\left(l^{2}+4 m\right) \rho^{2}}{16 l^{6}-8 l^{4} \rho+\left(l^{2}+4 m\right) \rho^{2}}\right)^{2}, \\
& \kappa^{2}\left\langle T_{a a}\right\rangle=\frac{\left(l^{2}+4 m\right)}{8 l} \cdot\left(\frac{16 l^{6}-16 l^{4} \rho+3\left(l^{2}+4 m\right) \rho^{2}}{16 l^{6}-\left(l^{2}+4 m\right) \rho^{2}}\right) \cdot w_{a a} .
\end{aligned}
$$

Note that this is the exact boundary energy momentum tensor at arbitrary boundary position $\rho=\rho_{0}$. Especially, at the UV fixed point $(\rho \rightarrow 0)$ the boundary energy $E=$ $V_{S^{3}}\left\langle T_{00}\right\rangle$ and the pressure $P_{a}=V_{S^{3}}\left\langle T_{a a} / w_{a a}\right\rangle$ describes boundary fields like massless gauge and scalar fields as a perfect fluid type matter having the equation of state parameter $\gamma=P / E=1 / 3$. When the boundary position is located at a finite distance $\rho_{0}$ which is equivalent to consider the boundary theory at the $\mathrm{RG}$ scale $\Lambda$, in this scale the interaction of the boundary fields can deform the equation of state parameter which may depend on the RG scale $\Lambda$ and the temperature $T=T_{H}$. Using the above the exact boundary energymomentum tensor, we can obtain the equation of state for the boundary field at arbitrary temperature and the RG scale, which can be written by, in terms of the bulk variables,

$$
\gamma(\rho, m)=\frac{\left(16 l^{6}-16 l^{4} \rho+3\left(l^{2}+4 m\right) \rho^{2}\right)\left(16 l^{6}-8 l^{4} \rho+\left(l^{2}+4 m\right) \rho^{2}\right)^{2}}{3\left(16 l^{6}-\left(l^{2}+4 m\right) \rho^{2}\right)^{3}} .
$$

The trace of this stress tensor is given by

$$
\kappa^{2}<T_{i}^{i}>=-\frac{24 l\left(l^{2}+4 m\right)\left(4 l^{4}-\left(l^{2}+4 m\right) \rho\right) \rho^{2}}{\left(16 l^{6}-\left(l^{2}+4 m\right) \rho^{2}\right)\left(16 l^{6}-8 l^{4} \rho+\left(l^{2}+4 m\right) \rho^{2}\right)} .
$$


In this global case, we can also write the stress tensor in a compact way which is proportional to the boundary metric :

$$
<T_{i j}>=C(\rho)<T_{i}^{i}>g_{i j}
$$

where

$$
C(\rho)=\frac{l^{2}}{4 \rho}-\frac{l^{2}\left(\rho-4 l^{2}\right)\left(1-4 \delta_{i 0} \delta_{j 0}\right)}{6 \rho^{2}\left[\pi^{2} T_{H}^{2}\left(l^{2} \pi^{2} T_{H}^{2}+l \pi T_{H} \sqrt{l \pi T_{H}-2}\right) \rho-2\right]} .
$$

The AdM mass is given by

$$
M=\lim _{\rho \rightarrow 0} \frac{3 \pi^{2}\left(l^{2}+4 m\right)}{64 \kappa^{2} l^{6}}\left|4 m \rho^{2}+l^{2} \rho^{2}-16 l^{6}\right|=\frac{3 \pi^{2} l^{2}}{4 \kappa^{2}}+\frac{3 m \pi^{2}}{\kappa^{2}} .
$$

The second term of the mass $M$ is the standard mass of SAdS black hole solution and the first term is the gravitational mass of global $A d S_{5}$ which is identified as the Casimir energy of $\mathrm{SU}(\mathrm{N}) \mathcal{N}=4 \mathrm{SYM}$ on $\mathbf{R} \times \mathbf{S}^{3}$ in the large $\mathrm{N}$ limit 9 , 21].

Using the holographic renormalization method, we can easily calculate the boundary stress tensor at the UV fixed point by inserting the metric (3.39) into (2.9)

$$
\kappa^{2}\left\langle T_{i j}^{0}\right\rangle=\operatorname{diag}\left(\frac{3\left(l^{2}+4 m\right)}{8 l^{3}}, \frac{\left(l^{2}+4 m\right)}{8 l}, \frac{\left(l^{2}+4 m\right)}{8 l} \sin ^{2} \theta, \frac{\left(l^{2}+4 m\right)}{8 l} \cos ^{2} \theta\right) .
$$

To compare the exact result with the above boundary stress tensor, we expand the exact one near $\rho=0$ and then the several terms of this are given by

$$
\begin{aligned}
\kappa^{2}\left\langle T_{00}\right\rangle & =\frac{3\left(l^{2}+4 m\right)}{8 l^{3}}+\frac{3\left(l^{2}+4 m\right) \rho}{8 l^{5}}+\frac{3\left(l^{2}-2 m\right)\left(l^{2}+4 m\right) \rho^{2}}{16 l^{9}}+\mathcal{O}\left(\rho^{3}\right), \\
\kappa^{2}\left\langle T_{a a}\right\rangle & =\left[\frac{\left(l^{2}+4 m\right)}{8 l}-\frac{\left(l^{2}+4 m\right) \rho}{8 l^{3}}+\frac{\left(l^{2}+4 m\right)^{2} \rho^{2}}{32 l^{7}}+\mathcal{O}\left(\rho^{3}\right)\right] w_{a a}, \\
\kappa^{2}\left\langle T_{i}^{i}\right\rangle & =-\frac{3\left(l^{2}+4 m\right) \rho^{2}}{8 l^{7}}-\frac{3\left(l^{4}-16 m^{2}\right) \rho^{3}}{32 l^{11}}-\frac{3\left(l^{4}-16 m^{2}\right) \rho^{4}}{64 l^{13}}-\mathcal{O}\left(\rho^{5}\right) .
\end{aligned}
$$

The leading term of the exact boundary stress tensor agrees with boundary stress tensor calculated in the holographic renormalization method, Eq. (3.46). The same result was founded by using an usual $r$-coordinate [9]. We may write the trace of the stress tensor in terms of the temperature of the black hole and the energy scale, as in the Poincare case. But in the case of the global patch, the resulting expression is very complicated and we omit it here. At the UV fixed point $\rho=0$, there is no trace anomaly as expected. Unlike the Poincare case, the trace anomaly defined on the boundary of the pure global AdS space-time $(m=0)$, has the $\rho$-dependence which is naturally coming in to describes the volume effect of the boundary space. In other words, the conformal symmetry near the UV fixed point is broken at the low energy scale because at this low energy scale the volume of the boundary space becomes finite. So this finite size effect break the conformal symmetry. For $m \neq 0$, the conformal symmetry is broken by the thermal effect as well as 


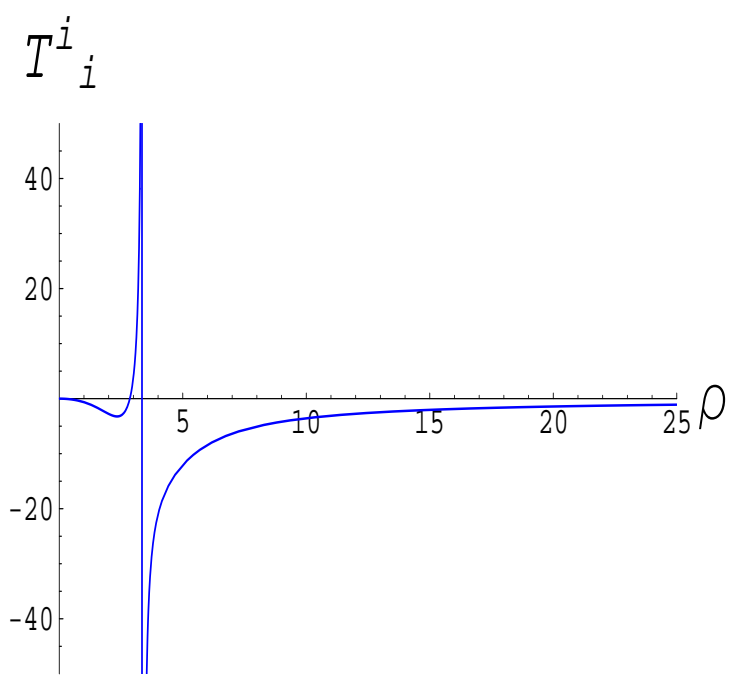

Figure 2: Trace Anomaly in the Global Patch. For convenience, we set $l=1$ and $\kappa^{2}=1$. With $m=0.1$, the trace anomaly is zero at $\rho_{f}=2.85714$ and diverges at the horizon $\rho_{h}=3.38062$.

the finite size effect. Interestingly, there exists a point $\rho_{f}=4 l^{4} /\left(l^{2}+4 m\right)$ where the finite size effect cancels the thermal effect so that the trace anomaly becomes zero at this point.

As previously mentioned in the introduction, in this paper we did not consider the logarithmic term of the boundary metric in the FG expansion and the ignorance of this term was justified by finding the exact solution in the FG coordinate. Following the references [10, 11], when considering the most general FG expansion including the logarithmic term, the metric variation of the trace anomaly at the UV fixed point $(\rho \rightarrow 0)$ comes from the coefficient of the logarithmic term in the FG expansion and the on-shell action with the trace anomaly picks up a logarithmic divergence [24]

$$
S_{\text {on-shell }} \approx \frac{l^{3}}{4 \kappa^{2}} \int d^{4} x\left(\operatorname{Tr}\left[g_{(0)}^{-1} g_{(2)} g_{(0)}^{-1} g_{(2)}\right]-\operatorname{Tr}\left[g_{(0)}^{-1} g_{(2)}\right] \operatorname{Tr}\left[g_{(0)}^{-1} g_{(2)}\right]\right) \ln \rho_{0} .
$$

In this case, using the FG expansion in Eq. (3.39), the coefficient of the logarithmic divergence term in the on-shell action is exactly zero, which is consistent with our exact solution for the black hole solution in Poicare and global patch. This is also consistent with the result of other paper [23] where it was found that the $\mathcal{N}=4$ SYM theory on the product space has no conformal anomaly.

\section{Discussion}

In this paper, we found the exact SAdS black hole solution in the FG coordinate. Although this describes the same well-known SAdS black hole solution, the exact one in FG coordinate gives much information for the boundary field theory. In the usual $r$-coordinate finding 
the induced metric on the boundary located at the finite distance is ambiguous because the metric component in the radial direction depends on the boundary position. In the FG coordinate, there is no such kind of problem since the metric component in the radial direction does not depend on the boundary position up to overall scale. Therefore, in the usual $r$-coordinate the induced boundary metric is well-defined only when the boundary is located at the infinity. To describe the Wilsonian RG of the boundary theory we need the exact solution in the FG coordinate. Here, using the exact black hole solutions in the FG coordinate we have calculated the trace anomaly of the boundary theory.

In the Poincare patch, since the boundary topology is $\mathbf{R} \times \mathbf{R}^{3}$ in the Mikowskian version (in the Euclidean version, $\mathbf{S} \times \mathbf{R}^{3}$ ), there is no scale except the black hole mass $m$. So when $m=0$, the boundary theory has always the conformal symmetry as the Wilsonian energy cut-off scale $\Lambda$ runs. Considering the black mass which gives rise to a scale, at the UV fixed point $\rho=0$ the boundary theory is still conformal but except this UV fixed point the conformal anomaly appears due to the black hole mass which can be reinterpreted as a thermal effect of the boundary theory.

In the case of the global patch, the boundary topology has $\mathbf{R} \times \mathbf{S}^{3}$ in the Minkowski space-time (in the Euclidean version, the topology is $\mathbf{S} \times \mathbf{S}^{3}$ ). After the scaling of the metric by $z^{2}$, the boundary topology of the spatial directions is given by $\mathbf{S}^{3}$ where the radial coordinate in the FG coordinate $z=\sqrt{\rho}$ plays a role of the radius of the three sphere. When the boundary is located at a finite distance of $\rho$, unlike the Poincare case, even for the pure AdS space-time $(m=0)$ there exists a trace anomaly due to the finite size effect of this three sphere. When considering the black hole solution in the global patch, there is another ingredient to break the conformal symmetry which is the thermal effect like in the Poincare case.

To obtain more insight for the duality between the gravity and gauge theory, it would be interesting to find the higher-dimensional asymptotically AdS space time solution in the FG coordinate and more non-trivial metric containing the effect of the other bulk fields. Furthermore, it is very interesting problem to evaluate the central charge depending on the temperature and the $\mathrm{RG}$ scale and to investigate the relation between the trace anomaly obtained here and these central charges. This may give us clues for understanding many interesting problems related to the RG flow like the c-theorem and the change of the black hole entropy by factor $3 / 4$ when going from strong to weak coupling regime.

\section{Acknowledgments}

We thank to Sang-Jin Sin, Ki-Myeong Lee, Ho-Ung Yee, Mu-in Park and Yunseok Seo for helpful discussion. This work was supported by the Science Research Center Program of 
the Korea Science and Engineering Foundation through the Center for Quantum Spacetime(CQUeST) of Sogang University with grant number R11 - 2005 - 021.

\section{References}

[1] J. M. Maldacena, "The large N limit of superconformal field theories and supergravity," Adv. Theor. Math. Phys. 2, 231 (1998) [Int. J. Theor. Phys. 38, 1113 (1999)] [arXiv:hep-th/9711200].

[2] S. S. Gubser, I. R. Klebanov and A. M. Polyakov, "Gauge theory correlators from non-critical string theory," Phys. Lett. B 428, 105 (1998) [arXiv:hep-th/9802109].

[3] E. Witten, "Anti-de Sitter space and holography," Adv. Theor. Math. Phys. 2, 253 (1998) [arXiv:hep-th/9802150].

[4] O. Aharony, S. S. Gubser, J. M. Maldacena, H. Ooguri and Y. Oz, "Large N field theories, string theory and gravity," Phys. Rept. 323, 183 (2000) [arXiv:hep-th/9905111].

[5] E. D'Hoker and D. Z. Freedman, "Supersymmetric gauge theories and the AdS/CFT correspondence," arXiv:hep-th/0201253.

[6] G. 't Hooft, "Dimensional reduction in quantum gravity," arXiv:gr-qc/9310026.

[7] L. Susskind, "The World As A Hologram," J. Math. Phys. 36, 6377 (1995) [arXiv:hep-th/9409089].

[8] J. D. Brown and J. W. . York, "Quasilocal energy and conserved charges derived from the gravitational action," Phys. Rev. D47, 1407 (1993).

[9] V. Balasubramanian and P. Kraus, "A stress tensor for anti-de Sitter gravity," Commun. Math. Phys. 208, 413 (1999) [arXiv:hep-th/9902121].

S. Nojiri and S. D. Odintsov, "Conformal anomaly for dilaton coupled theories from AdS / CFT correspondence," Phys. Lett. B444 92 (1998); S. Nojiri and S. D. Odintsov, "Conformal anomaly from dS / CFT correspondence," Phys. Lett. B519 145 (2001); S. Nojiri and S. D. Odintsov, "Universal features of the holographic duality: Conformal anomaly and brane gravity trapping from 5-D AdS black hole," Int. J. Mod. Phys. A18 2001 (2003).

[10] S. de Haro, S. N. Solodukhin and K. Skenderis, "Holographic reconstruction of spacetime and renormalization in the AdS/CFT correspondence," Commun. Math. Phys. 217, 595 (2001) [arXiv:hep-th/0002230].

[11] M. Henningson and K. Skenderis, "Holography and the Weyl anomaly," Fortsch. Phys. 48, 125 (2000) [arXiv:hep-th/9812032].

M. Henningson and K. Skenderis, "The holographic Weyl anomaly," JHEP 9807, 023 (1998) [arXiv:hep-th/9806087].

[12] C. Fefferman and C. Robin Graham, "Conformal invariants," The mathematical heritage of Elie Cartan (Lyon, 1984). Asterisque 1985, Numero Hors Serie, 95-116. 
[13] C. R. Graham, "Volume and area renormalizations for conformally compact Einstein metrics," [arXiv:math.dg/9909042].

[14] C. R. Graham and E. Witten, "Conformal anomaly of submanifold observables in AdS/CFT correspondence," Nucl. Phys. B546, 52 (1999) [arXiv:hep-th/9901021].

[15] I. R. Klebanov, "TASI lectures: Introduction to the AdS/CFT correspondence," arXiv:hep-th/0009139.

[16] L. Susskind and E. Witten, "The holographic bound in anti-de Sitter space," arXiv:hep-th/9805114.

[17] J. de Boer, E. P. Verlinde and H. L. Verlinde, "On the holographic renormalization group," JHEP 0008, 003 (2000) [arXiv:hep-th/9912012]

E. P. Verlinde and H. L. Verlinde, "RG-flow, gravity and the cosmological constant," JHEP 0005, 034 (2000) [arXiv:hep-th/9912018]

J. de Boer, "The holographic renormalization group," Fortsch. Phys. 49, 339 (2001) [arXiv:hep-th/0101026].

[18] C. Imbimbo, A. Schwimmer, S. Theisen and S. Yankielowicz, "Diffeomorphisms and holographic anomalies," Class. Quant. Grav. 17, 1129 (2000) [arXiv:hep-th/9910267].

A. Schwimmer and S. Theisen, "Diffeomorphisms, anomalies and the Fefferman-Graham ambiguity," JHEP 0008, 032 (2000) [arXiv:hep-th/0008082].

A. Schwimmer and S. Theisen, "Universal features of holographic anomalies," JHEP 0310, 001 (2003) [arXiv:hep-th/0309064].

[19] S. Nakamura and S. J. Sin, "A holographic dual of hydrodynamics," JHEP 0609, 020 (2006) [arXiv:hep-th/0607123].

[20] R. C. Myers, "Stress tensors and Casimir energies in the AdS/CFT correspondence," Phys. Rev. D 60, 046002 (1999) [arXiv:hep-th/9903203].

[21] G. T. Horowitz and R. C. Myers, "The AdS/CFT correspondence and a new positive energy conjecture for general relativity," Phys. Rev. D 59, 026005 (1999) [arXiv:hep-th/9808079].

[22] S. S. Gubser, I. R. Klebanov and A. W. Peet, "Entropy and Temperature of Black 3-Branes," Phys. Rev. D 54, 3915 (1996) [arXiv:hep-th/9602135].

[23] C. P. Burgess, N. R. Constable and R. C. Myers, "The free energy of N = 4 superYang-Mills and the AdS/CFT correspondence," JHEP 9908, 017 (1999) [arXiv:hep-th/9907188].

[24] R. Emparan, C. V. Johnson and R. C. Myers, Phys. Rev. D 60, 104001 (1999) [arXiv:hep-th/9903238]. 